\title{
Characteristics of a microbial consortium with high lignocelluloses-decomposing capacity
}

\author{
Guo-xiang ZHENG ${ }^{1,2 *}$, Chen-yang $\mathrm{ZHOU}^{1}$ and Jian $\mathrm{LI}^{1}$ \\ ${ }^{1}$ College of Engineering, Northeast Agricultural University, Harbin 150030, China \\ ${ }^{2}$ Key Laboratory of Pig-breeding Facilities Engineering, Ministry of Agriculture, Harbin 150030, \\ China \\ ${ }^{3}$ Heilongjiang Key Laboratory of Technology and Equipment for the Utilization of Agricultural \\ Renewable Resources, Harbin 150030, China \\ azgx720331@126.com; ${ }^{b} 18088701183 @ 163 . c o m ;{ }^{\circ} \mathrm{mlrs} 2345 @ 163 . c o m$
}

Keywords: Microbial consortium, degradation capacity, end metabolism product

Abstract. A microbial consortium, designated LZF-12, was obtained by successive subcultivation in the presence of rice straw under static conditions at $35^{\circ} \mathrm{C}$. The number of bacilli was dominant in microbial consortium by scanning electron microscopy. The degradation efficiencies of LZF-12 for rice straw, hemicelluloses, cellulose and lignin were $70 \% 、 92.9 \%, 82.6 \%$ and $21.8 \%$, respectively for 12 days cultivation. There was near $70 \%$ of acetic acid in end metabolism products in addition to little butyric acid, propionic acid and ethanol. This fermentation type will provide essential material base for stable and effective operation of methanogenic phase in two-phase anaerobic digestion process.

\section{Introduction}

Lignocellulosic wastes are the most abundant and environment-friendly material generated annually because of forestry, agricultural and industrial activities [1]. With the depletion of non-renewable energy, as the most attractive and promising avenue for cost-efficient treatment, bioconversion of large amounts of lignocellulosic biomass into fermentable material has been considered to be potential application in the area of bioenergy generation [2]. Therefore, enormous research efforts have been invested on bioconversion of lignocellulosic biomass in decades [3, 4]. However, low degradation efficiency has been a major challenge for lignocellulosic waste biodegradation due to the complex stable structure of lignocellulosic biomass [5]. As we known, cellulosic bioconversion is a multi-step process requiring a multi-enzyme complex generated by complex microbial communities for efficient bioconversion into added-products [6]. In nature, it is more difficult for single strains to degrade lignocellulosic biomass due to the refractory structure of cellulose and feedback regulation and metabolite repression [7]. Compared with single-species systems, microbial co-cultures or complex communities has been considered the most promising and highly efficient approach for the biodegradation of lignocellulose wastes, and microbial consortium play more better synergic role in the cellulose-degrading systems. More complex microbial systems with good lignocellulose decomposition ability were screened out from different environment $[8,9,10,11]$.

In this study, a microbial consortium capable of degrading lignocellulosic biomass was enriched from successive enrichment cultures on PCS (peptone cellulose solution) medium at $35^{\circ} \mathrm{C}$. Study on degradation characteristic of microbial consortia was investigated, which could get an insight into the dynamic process of bioconversion and also provide an important basis for understanding the potential of lignocellulose degradation in subsequent effective operation of acidification.

\section{Materials and methods}

\section{Materials}

Rice straw was obtained from local farm (Harbin, China). Filter paper was purchased from Xinhua paper Corporation (Hangzhou, China). Rice straw was cut into about $1.0 \mathrm{~cm}$ in length and soaked in 
$1 \% \mathrm{NaOH}$ for $24 \mathrm{~h}$, washed with distilled water until neutrality and dried at $60{ }^{\circ} \mathrm{C}$. Cellulosic materials were autoclaved at $121^{\circ} \mathrm{C}$ for $15 \mathrm{~min}$ before use. All chemicals and reagents used in this work were of analytical or molecular biology grade. Chemicals and reagents were analytical or molecular biology grade.

\section{Isolation of lignocellulolytic microbial consortium}

The lignocellulose degrading microbial consortium was bred from 24 original soil sample from swamp and perennial deciduous forest humus. a $1 \mathrm{~g}$ soil sample was inoculated into a $100 \mathrm{ml}$ screw-cap disposable tube containing $50 \mathrm{ml}$ of PCS medium $\left(0.1 \%\right.$ yeast extract, $0.5 \%$ peptone, $0.3 \% \mathrm{CaCO}_{3}$, $0.5 \% \mathrm{NaCl}, 1 \%$ rice straw) with a filter paper strip as an indicator for cellulase activity. The mixture was incubated at $35^{\circ} \mathrm{C}$ under static facultative anaerobic conditions. Those samples in which the disintegration time of filter paper exceeds 7 days and $\mathrm{pH}$ descends slowly were eliminated. The remaining 2 groups were continuously subcultivated for over 30 generations to investigate their genetic stability. A structurally stable composite system named LZF-12 with high disintegration capacity was obtained and maintained in PCS medium containing $20 \%$ glycerol at $-80{ }^{\circ} \mathrm{C}$ for long -term storage or subsequent experimental studies.

\section{analysis methods}

The microbial consortium was incubated in PCS medium containing 1\% rice straw. Residual solid cellulosic substrates were analyzed after incubation with the microbial consortium for 12 days at $35^{\circ} \mathrm{C}$ under the defined facultative anaerobic static conditions. The residual substrates were then determined gravimetrically after drying at $105^{\circ} \mathrm{C}$ for $72 \mathrm{~d}$ with uninoculated medium as a control. \% Residual weight was reported based on total holocellulose content [1]. The reactions were performed in triplicate and the averages of the results were reported.

Cellulose, Hemicellulose, lignin and VFAs were determined as described by Liu [11]. VFAs was determined by a gas chromatograph (GC-6890N, Agilent Inc., USA) equipped with a flame ionization detector. DO and $\mathrm{pH}$ were measured using portable dissolved oxygen analyzer (oxi315i, WTW, Germany) and acidity /ORP/ ion measuring analyzer (HI253A, HANNA, Italy), respectively. The morphological structure of microbial consortium and rice straw was determined by scanning electron microscope (SEM) (S-3400N, Hitachi, Japan's).

\section{Results and discussion}

\section{Stability of microbial consortium LZF-12}

Lignocellulose degradation rate, $\mathrm{pH}$, dissolved oxygen (DO) and liquid metabolites were used as indicators to monitor LZF-12's fermentation and growth performance. After investigating genetic stability of microbial community by serials intermittent tests, microbial consortium LZF-12 was proved to be a facultative anaerobe group and show a strongly relative stability. As shown in Table 1, in the end of fermentation, dissolved oxygen maintain at $0.05 \sim 0.10 \mathrm{mg} / \mathrm{L}$ in different generation's system, suggesting microbial consortium LZF-12 was a group of facultative bacteria, which this microaerobic environment is more suitable to the decomposition of lignocelluloses [10]. Seen from final $\mathrm{pH}$, value showed slight fluctuation from 7.65-8.01, indicating relative better self-regulation ability. The maximum acetic acid concentrations was measured from 1.83 to $2.18 \mathrm{mg} / \mathrm{L}$ of different generation's cultures in end liquid metabolism, which accounted for more than $70 \%$ of the total volatile acid, suggesting it will be more conducive to utilization by methanogenic bacteria of the follow-up biogas production phase. Experimental data also indicated a total loss of rice straw ranged from $65 \% \sim 70 \%$ in 15-40 generation cultures system ( Table 1 ), demonstrating the microbial consortium LZF-12 had strong ability to degrade cellulose and genetic stability, which might provide important material basis for follow-up application and synergistic metabolic mechanism. 
Table 1. The inherit stability of composite microbial system

\begin{tabular}{|c|c|c|c|c|}
\hline Generations & DO/ ( mg/L) & Final pH & $\begin{array}{c}\text { Maximum amount of } \\
\text { acetic acid }(\mathrm{mg} / \mathrm{L})\end{array}$ & $\begin{array}{c}\text { the loss rate of straw } \\
\text { weight }(\%)\end{array}$ \\
\hline 15 & $0.06 \sim 0.1$ & 7.75 & 1.89 & 68.1 \\
20 & $0.07 \sim 0.09$ & 7.65 & 2.18 & 69.0 \\
25 & $0.06 \sim 0.09$ & 7.78 & 2.04 & 68.6 \\
30 & $0.05 \sim 0.08$ & 8.01 & 1.88 & 70.3 \\
35 & $0.06 \sim 0.09$ & 7.88 & 1.83 & 69.7 \\
40 & $0.05 \sim 0.1$ & 7.86 & 1.90 & 67.1 \\
\hline
\end{tabular}

Structure of the rice straw and the morphological characteristics of the microbial community

In this study, the changes of surface structure of rice stem were observed by scanning electron microscope (SEM). Seen from Fig.1, Untreated straw, irrespective of inner or outer surfaces, showed regular, tight and compact feature (Fig.1-A), due to compact high-level structure, cellullases secreted by microorganisms are difficult to contact with cellulose and directly affects subsequent hydrolysis and fermentation in rice straw digestion process [12]. By comparison, the surface of rice straw treated by $1 \% \mathrm{NaOH}$ for $24 \mathrm{~h}$ appeared obvious breakdown and part of fibre exposed, this will create relatively favorable conditions for microbial degradation of straw (Fig.1-B). Fig.1-C recorded the morphological structural variation of rice straw, significant degradable components and debris has presented by microbial community LZF-12 treating 5days, dominant brevi bacteria spread in straw fibers, demonstrating LZF-12 used degradable and digestible components of rice straw to make a large number of propagation.
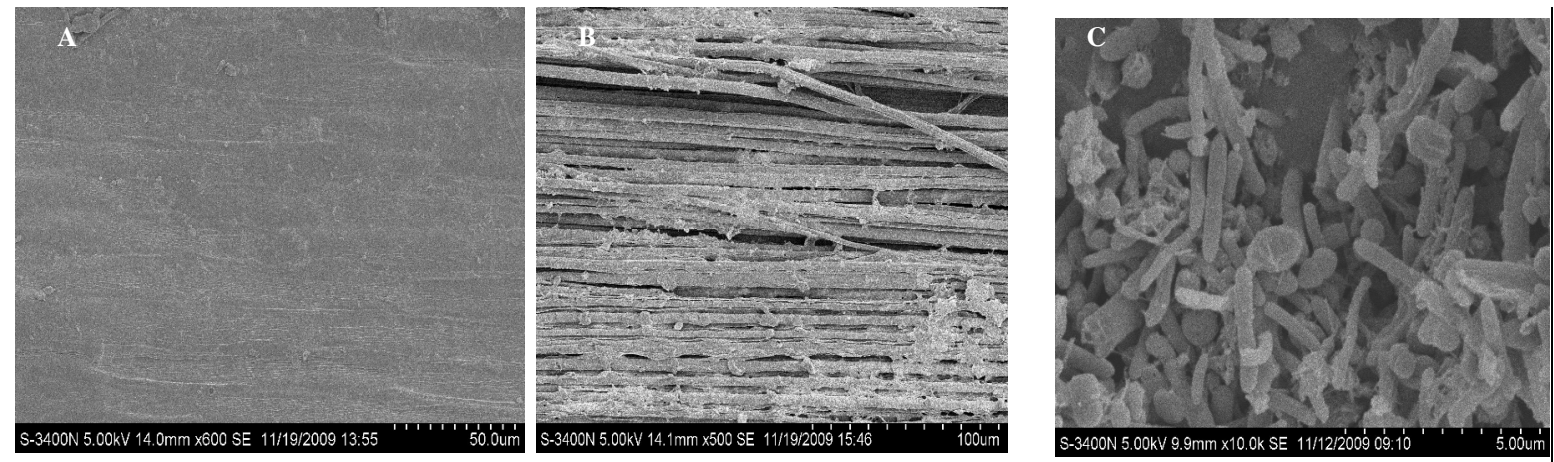

A: Inner surface of original straw without treatment by alkali B: Inner surface of straw treated by alkali C: Inner surface of straw treated 5 days by microbial community LZF-12

Figure 1 Change of micro-appearance of rice straw

\section{Degradation performance of microbial community LZF-12}

As shown in Fig.2, there appeared a lot variation on ingredients of rice straw degraded by microbial system LZF-12. The content of hemicellulose, cellulose, lignin were $0.42 \mathrm{~g}, 0.92 \mathrm{~g}, 0.064 \mathrm{~g}$ respectively using alkali-treated rice straw, and concentration of hemicellulose decreased from initial $0.42 \mathrm{~g}$ down to $0.12 \mathrm{~g}$, within $0 \sim 50 \mathrm{~h}$ after inoculated with LZF-12, then showed slow degradation in $50 \sim 300 \mathrm{~h}$. When fermentation time reached $50 \mathrm{~h}$, hemicellulose concentration was dropped to $0.03 \mathrm{~g}$, the total degradation rate was $92.9 \%$ at the end of fermentation. Whereas a slight change was measured in cellulose content in $0-50 \mathrm{~h}$, its value was only $0.3 \%$, then cellulose concentration declined rapidly and the total cellulose degradation reached $81.8 \%$ in $50 \sim 300 \mathrm{~h}$. As this can be determined, the microbial system LZF-12 first use easily degradable hemicelluloses, then decompose cellulose rapidly, indicating the complexity of cellulose structure and not easily degradable characteristic by microorganisms. Compared to cellulose and hemicelluloses, lignin content (Fig.2) showed little variation due to special complex structure composed of guaiacyl, lilac and p-hydroxyphenyl to be difficult to degrade by microbial system. 


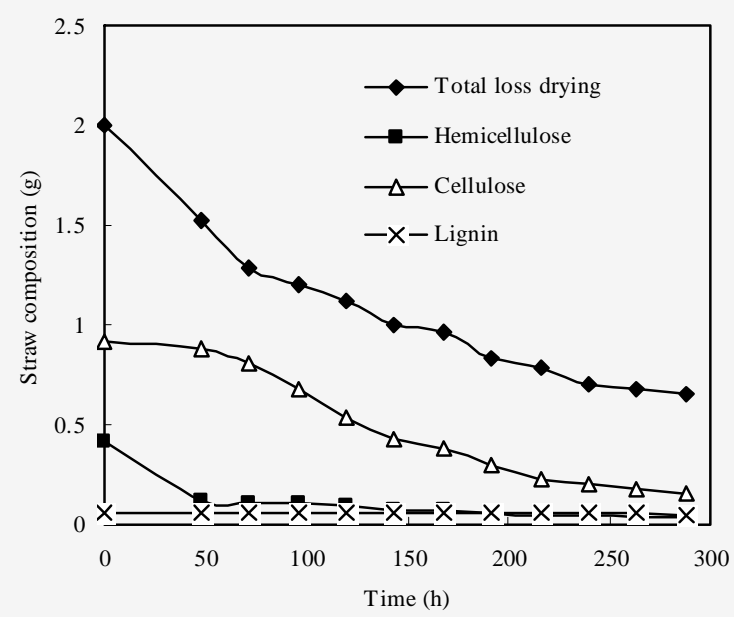

Figure. 2 Changes of rice straw composition during degradation

[13].As shown in Fig.3, a significant amount of VFAs was produced during degradation of cellulose by microbial consortium LZF-12. Similar change trend of acetic acid, propionic acid and butyric acid was observed that first increased then decreased with time. The maximum values $2.38 \mathrm{~g} / \mathrm{L}$ of acetic acid and $3.43 \mathrm{~g} / \mathrm{L}$ of total amount of volatile acid were obtained in $125 \mathrm{~h}$, respectively, the amount of acetic acid accounted for near $70 \%$ of total soluble metabolite with fewer butyric acid, propionic acid and ethanol, which was suitable substrate for sub-sequent bioconversion by methanogenic bacteria in biogas-producing phase.
Total weight loss of rice straw included the degradation of cellulosic materials and non-cellulosic materials. In plant tissue, in addition to cellulose, hemicellulose, lignin, there are a lot of other material such as ash, pectin, protein and other components which occupy a considerable proportion (about 30\%)[2]. Figure. 2 showed that there was still about $30 \%$ undegraded material in rice straw at the end of the fermentation; main ingredient was $80 \%$ ash, which is hard to biotransformation but can be effectively utilized.

\section{Degradation end liquid products}

Volatile acid are the end product of hydrolysis acidification, their varieties and output are very important for the next methanogenic phase

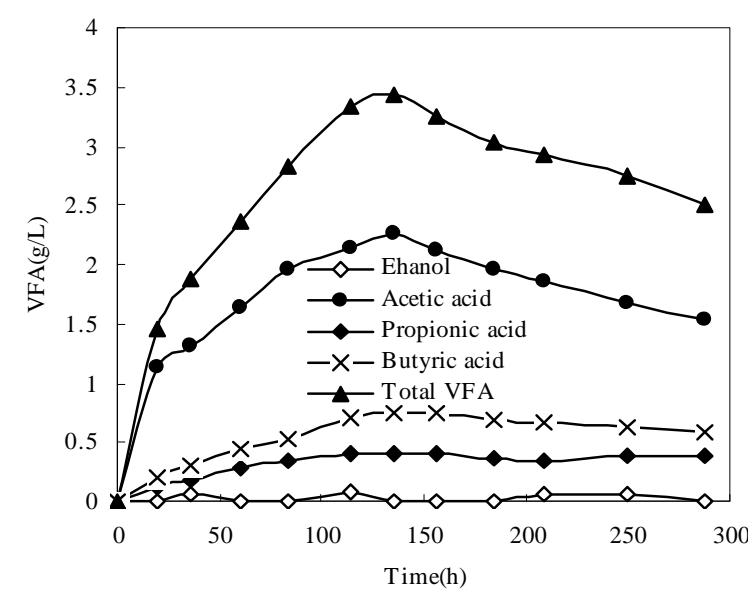

Figure. 3 The curse of liquid end products during fermentation

\section{Conclusion}

As we known, the utilization of lignocellulosic biomass for biofuel production is a renewable alternative with the increasing demands for energy and the shrinking energy resources [14]. A microbial consortium LZF-12 with good degradable lignocellulose performance was obtained by means of a succession of enrichment cultures and bacilli were determined to be main predominant bacteria by scanning electron microscope. After bioconversion by LZF-12, degradation rate of hemicelluloses, cellulose and lignin in rice straw reached $92.9 \%, 82.6 \%$ and $21.8 \%$, respectively, by tracking components changes of rice straw. Total straw weight loss exceeds $70 \%$ in $288 \mathrm{~h}$ at $35^{\circ} \mathrm{C}$, suggesting microbial consortium LZF-12 has a relative strong lignocelluloses-decomposing capacity. In addition, total amount of acetic acid and butyric acid accounting for more than $90 \%$ of total end metabolism was observed in LZF-12 lignocelluloses-decomposing system, their abundance may be beneficial to the growth of methanogenic bacteria in follow-up biogas-production stage in two phase anaerobic digestion system. It will also provide important bacterial species resources for implementation strategy of reinforcement acidification phase.

\section{Acknowledgement}

This research was financially supported by the National Science Foundation of Heilongjiang Province (E2015023) and Postdoctoral Launch Fund of Heilongjiang Province (LBH-Q13023) 


\section{References}

[1] S. Wongwilaiwalina, U. Rattanachomsria, T. Laothanachareona, et al., Analysis of a thermophilic lignocellulose degrading microbial consortium and multi-species lignocellulolytic enzyme system , Enzyme and Microbial Technology, 47 (2010) 283- 290.

[2] R. Kumar, S. Singh, O. V. Singh. Bioconversion of lignocellulosic biomass: biochemical and molecular perspectives, J Ind Microbiol Biotechnol. 35 (2008) 377-391.

[3] X. L. Han, W. X. Song, G. D. Liu et al., Improving cellulase productivity of Penicillium oxalicum RE-10 by repeated fed-batch fermentation strategy. Bioresource Technology. 227 (2017) 155-163.

[4] W.D. Wang, L. Yan, Z. J. Cui, et al., Characterization of a microbial consortium capable of degrading lignocellulose, Bioresource Technology. 102 (2011) 9321-9324.

[5] L.Yu, Z. X. Cen, X. Tong, et al., Anaerobic degradation of microcrystalline cellulose: Kinetics and micro-scale structure evolution, Chemosphere. 86 (2012) 348- 353.

[6] Y. X. Chen, S. K. Chang, J. Chen, et al., Characterization of microbial community succession during vermicomposting of medicinal herbal residues, Bioresource Technology. 249 (2018) 542-549.

[7] A. J. Ragauskas, C. K. Williams, B. H. Davison, et al., The path forward for biofuels and biomaterials, Science 311(2006):484- 489.

[8] P. Guo, W. B. Zhu, H. Wang, et al., Functional characteristics and diversity of a novel lignocelluloses degrading composite microbial system with high xylanase activity, Journal of Microbiology and Biotechnology. 20(2010) 254-264.

[9] S. Haruta, Z. Cui, Z. Huang, M. Li, et al., Construction of a stable microbial community with high cellulose-degradation ability, Appl Microbiol Biotechnol. 59 (2002) 529- 534.

[10] S. Kato, S. Haruta, Z. J. Cui, et al., Stable coexistence of five bacterial strains as a cellulose-degrading community, Appl Environ Microbiol. 71 (2005) 7099- 7106.

[11] Z. D. Liu, W. Z. Li, S. Liu, et al., Characteristic of an efficient microbial system with cellulose degradation bacteria. Journal of Northeast Agricultural Univeristy, 8 (2009): 105-109 (China).

[12] D Tam-Anh, S. Nguyena, H. Jong. Hydrogen production by the hyperthermophilic eubacterium, Thermotoga neapolitana, using cellulose pretreated by ionic liquid, International journal of hydrogen energy. 33(2008): 5161-5168.

[13] Y. T. Zhong, W. Z. Li, G. X. Zheng. Screening of rice straw degradation microbial system and its growth characteristics. Journal of northeast agricultural university. 8(2007):56-61. (China)

[14] D. Antoni, V. V. Zverlov, W. H. Schwarz Biofuels from microbes. Appl Microbiol Biotechnol 77(2007):23-35. 